\title{
Enhanced Charge Transport and Incorporation of Redox Mediators in Layer-by-Layer Films Containing PAMAM-Encapsulated Gold Nanoparticles
}

\author{
Frank N. Crespilho, ${ }^{\dagger,}$ Valtencir Zucolotto, ${ }^{\S}$ Christopher M. A. Brett, \\ Osvaldo N. Oliveira, Jr., $\$$ and Francisco C. Nart*,† \\ Instituto de Química de São Carlos and Instituto de Física de São Carlos, Universidade de São Paulo, \\ 13560-970 Brazil, and Departamento de Química, Universidade de Coimbra, 3004-535 Coimbra, Portugal
}

Received: April 4, 2006; In Final Form: May 23, 2006

\begin{abstract}
In this work, we exploit the molecular engineering capability of the layer-by-layer (LbL) method to immobilize layers of gold nanoparticles on indium tin oxide (ITO) substrates, which exhibit enhanced charge transfer and may incorporate mediating redox substances. Polyamidoamine (PAMAM generation 4) dendrimers were used as template/stabilizers for Au nanoparticle growth, with PAMAM-Au nanoparticles serving as cationic polyelectrolytes to produce LbL films with poly(vinylsulfonic acid) (PVS). The cyclic voltammetry (CV) of ITO-PVS/PAMAM-Au electrodes in sulfuric acid presented a redox pair attributed to Au surface oxide formation. The maximum kinetics adsorption is first-order, 95\% of the current being achieved after only 5 min of adsorption. Electron hopping can be considered as the charge transport mechanism between the PVS/ PAMAM-Au layers within the LbL films. This charge transport was faster than that for nonmodified electrodes, shown by employing hexacyanoferrate(III) as the surface reaction marker. Because the enhanced charge transport may be exploited in biosensors requiring redox mediators, we demonstrate the formation of Prussian blue (PB) around the Au nanoparticles as a proof of principle. PAMAM-Au@PB could be easily prepared by electrodeposition, following the ITO-PVS/ PAMAM-Au LbL film preparation procedure. Furthermore, the coverage of $\mathrm{Au}$ nanoparticles by $\mathrm{PB}$ may be controlled by monitoring the oxidation current.
\end{abstract}

\section{Introduction}

Dendrimers are a special class of organic molecules that can undergo a series of chemical modifications through surface chemistry while their interior cavities serve as templates for nanoparticle growth. Polyamidoamine (PAMAM) dendrimers, in particular, have been recently reported as very effective nanoparticle stabilizers, allowing their use as nanoreactors. ${ }^{1-7}$ Dendrimer molecules possess three basic architectural components: an initiator core (e.g., ethylenediamine), interior layers often called "generations", which comprise repeating units attached to the initiator core, and the shell which generally consists of functionalized groups attached to the outermost interior layer. It is well-known that dendrimers of lower generations tend to exist in relatively open forms, while high generation dendrimers take on a spherical three-dimensional structure. The latter structure, however, is very different from conventional linear polymers, which adopt random-coil structures. Thus, many interesting reactions using dendrimers have been reported because they can provide reaction sites in the interior or on the periphery of the dendrimers and are very useful as model systems. ${ }^{8-20}$

Monolayers of PAMAM dendrimers have been prepared by electrostatic self-assembly from aqueous solutions. ${ }^{9,10}$ Bliznyuk et al. proposed a model of molecular ordering, which assumes highly deformed (compressed parallel to the surface) ellipsoidal shapes for macromolecules in condensed monolayers. The molecular dimension (thickness) versus molecular mass depen-

\footnotetext{
* To whom corespondence should be addressed. E-mail: nart@iqsc.usp.br

† Instituto de Química de São Carlos, Universidade de São Paulo.

$\div$ Universidade de Coimbra.

§ Instituto de Física de São Carlos, Universidade de São Paulo.
}

dence is described by a scaling law. ${ }^{9}$ Cheng and Cox employed the layer-by-layer ( $\mathrm{LbL}$ ) deposition technique to fabricate multilayer films consisting of polyoxometalates (POMs) and G4-PAMAM. ${ }^{10}$ They demonstrated the fabrication of uniform and well-defined multilayered supramolecular structures consisting of inorganic POMs and organic PAMAM for application in catalysis. Hybrid nanoparticles of carboxyl-terminated PAMAM dendrimers containing encapsulated Pt nanocrystals were prepared, and reports on the fabrication of $\mathrm{Au}, \mathrm{Cu}$, and $\mathrm{Pd}$ nanoparticles within PAMAM dendrimers ${ }^{8}$ have also been made. Highly monodispersed, 1-2 nm diameter, Au nanoparticles were prepared by Kim et al. ${ }^{22}$ using PAMAM dendrimers as templates (PAMAM-Au). The synthesis was carried out in water and took less than $30 \mathrm{~min}$, requiring no subsequent purification. He et al. reported the electrostatic LbL assembly of a Au-dendrimer nanocomposite using poly(sodium 4-styrenesulfonate) (PSS) as the oppositely charged polyelectrolyte leading to nanoscale uniform multilayers of Au-dendrimer nanoclusters.

An advantage of PAMAM nanoreactors is the small nanoparticle diameter. Metal nanoparticles (less than $4 \mathrm{~nm}$ in diameter) are interesting because of their inherent size-dependent optical, electrical, catalytic, and magnetic properties. ${ }^{11-16}$ These materials have been integrated into new kinds of biosensors, have shown effects of particle size on heterogeneous catalytic reactions, and have been used for the fabrication of nanometerscale electronic devices, supercapacitors, and data storage devices. ${ }^{11-22}$

In this work, we report the fabrication of nanostructured films comprising nanoparticle-containing amine-terminated G4 PAMAM dendrimer and poly(vinylsulfonic acid) (PVS) through the LbL technique. Nanosized Au nanoparticles were grown inside PAMAM molecules using formic acid as the reduction agent. 
SCHEME 1: Schematic Fabrication of LbL Films Comprising PVS and Gold Nanoparticles Encapsulated in Polyamidoamine Generation 4 (G4 PAMAM) Dendrimers $^{a}$

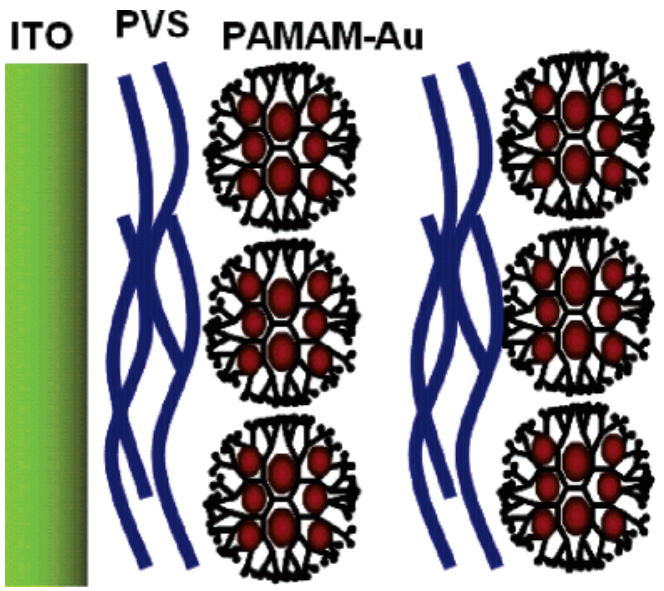

${ }^{a}$ The sequential deposition of LbL multilayers was carried out by immersing the substrates alternately into the PAMAM-Au and PVS solutions for $5 \mathrm{~min}$. After deposition of each layer, the substrate/film system was rinsed and dried with $\mathrm{N}_{2}$.

The PAMAM-Au hybrids were then assembled onto an indium tin oxide (ITO) electrode for electrochemical characterization. The aim was to obtain enhanced charge transport, which may be important for a number of applications, especially in biosensors requiring redox mediators. To further exploit the enhanced transport, in subsequent electrochemical experiments we show that a new system based on Prussian blue (PB) around the Au nanoparticles (PAMAM-Au@PB) can be easily obtained by electrodeposition using cyclic voltammetry $(\mathrm{CV})$.

\section{Experimental Section}

Synthesis and Characterization of PAMAM-Au. AuPAMAM nanohybrids were prepared as follows: $2 \mathrm{~mL} \mathrm{KAuCl}_{4}$ solution $\left(1 \mathrm{mmol} \mathrm{L}^{-1}\right)$ was added to $2 \mathrm{~mL}$ PAMAM $(0.07 \mathrm{mmol}$ $\left.\mathrm{L}^{-1}\right)$ and $2 \mathrm{~mL}$ formic acid $\left(1 \mathrm{mmol} \mathrm{L}^{-1}\right)$. This pale yellow solution was vigorously stirred for $2 \mathrm{~min}$. When the zerovalent Au complex was formed, the color immediately changed from yellow to red. This reaction occurred over a $4 \mathrm{~h}$ time period, and the nanoparticle growth kinetics was followed by UV-vis spectroscopy (Hitachi U-2001 spectrophotometer; San Jose, CA). The morphology and particle size distribution were characterized using a $200-\mathrm{kV}$ transmission electron microscope (TEM, model CM200; Philips, Eindhoven, The Netherlands). The particle size distribution was estimated by the measurement of at least 200 particles in TEM images.

PVS/PAMAM-Au Multilayer Self-Assembly. PVS/PAMAM$\mathrm{Au} \mathrm{LbL}$ and cast films were assembled onto hydrophilic glass, quartz, ITO-coated glass, and silicon substrates. The concentration of the dipping solutions was set at $0.07 \mathrm{mmol} \mathrm{L}^{-1}$ and 0.5 $\mathrm{g} \mathrm{L}^{-1}$ for PAMAM-Au and PVS, respectively. The sequential deposition of multilayers was carried out in an HMS series programmable slide stainer (Carl Zeiss Inc, Jena, Germany) by immersing the substrates alternately into the PAMAM-Au and PVS solutions for $5 \mathrm{~min}$. After deposition of each layer (Scheme 1 ), the substrate/film system was rinsed and dried with $\mathrm{N}_{2}$. The growth of the multilayers was monitored by cyclic voltammetry. Fourier transform infrared spectroscopy (FTIR) measurements were carried out on films deposited onto Si substrates using a Nicolet 470 Nexus spectrometer (Madison, WI), after the sample chamber had been purged with $\mathrm{N}_{2}$ gas.
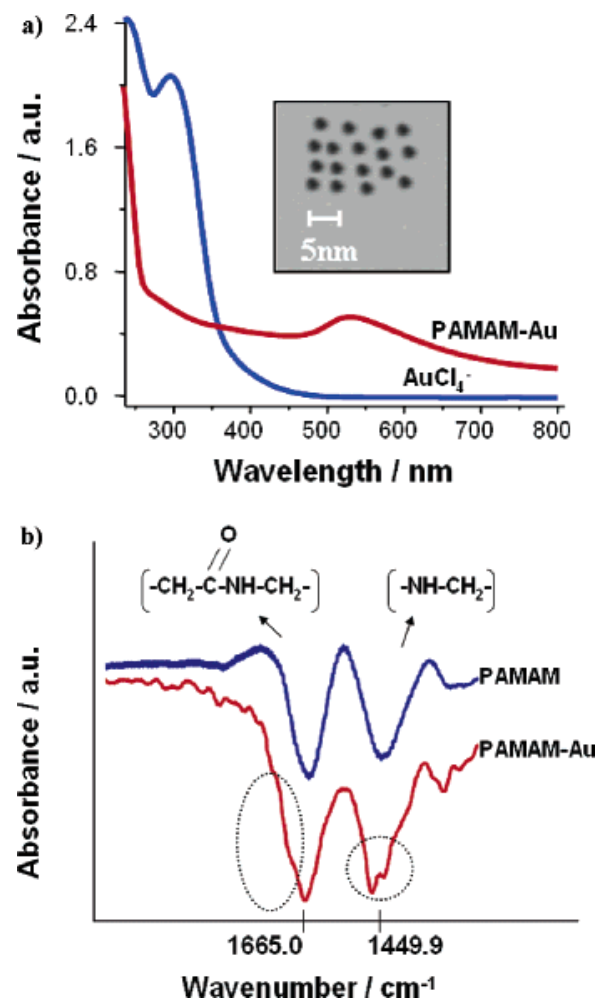

Figure 1. (a) Electronic absorption spectra of $\mathrm{KAuCl}_{4}$ solution (blue line) and PAMAM-Au suspension (red line). Inset: TEM images of a layer of PAMAM-Au nanoparticles with resolution of $5 \mathrm{~nm}$. (b) FTIR spectra for PAMAM and PAMAM-Au films deposited onto $\mathrm{Si}$ substrates.

Cyclic Voltammetry. Cyclic voltammograms (obtained in films containing different numbers of bilayers) were registered using on Autolab type II running with GPES (General Purpose Electrochemical System) 4.9 software (EcoChemie, Utrecht, The Netherlands). The electrochemical cell was a three-electrode system. $\mathrm{A} \mathrm{Ag} / \mathrm{AgCl}$ (sat. $\mathrm{KCl}$ ) electrode was used as reference, $\mathrm{Pt}$ wire as counter electrode, and ITO or ITO covered with a $(\mathrm{PVS} / \mathrm{PAMAM}-\mathrm{Au})_{n}$ film as the working electrode (where $n$ is the number of PVS/PAMAM-Au bilayers). All electrodes were inserted into the electrochemical cell containing a $\mathrm{H}_{2} \mathrm{SO}_{4}(0.5$ mol $\mathrm{L}^{-1}$ ) solution, and the voltammogram was immediately recorded. The voltammograms were recorded at $293 \mathrm{~K}$ at different scan rates. Before each cycle, the electrolyte was purged with $\mathrm{N}_{2}$ gas for $15 \mathrm{~min}$. The charge-transfer mechanism was studied using $5 \mathrm{mmol} \mathrm{L}^{-1} \mathrm{Fe}(\mathrm{CN})_{6}{ }^{-3}$ as electroactive species in $0.5 \mathrm{~mol} \mathrm{~L}^{-1} \mathrm{H}_{2} \mathrm{SO}_{4}$ electrolyte solution.

\section{Results and Discussion}

Characterization of the Au Nanoparticles. The formation of the $\mathrm{Au}$ nanoparticles inside PAMAM was monitored via UV-vis spectroscopy, showing a time-dependent spectrum for the reduction of $\mathrm{Au}(\mathrm{III})$ to $\mathrm{Au}(0)$ and growth of nanosize $\mathrm{Au}$ particles. The initial reduction stage may be followed by the decrease in the $300 \mathrm{~nm} \mathrm{Au(III)} \mathrm{band,} \mathrm{as} \mathrm{shown} \mathrm{in} \mathrm{Figure} \mathrm{1a.}$ The peak around $500 \mathrm{~nm}$ is associated with the plasmon resonance. An increase in the baseline due to scattering effects is also observed. ${ }^{1}$ For the conditions employed, the complete reduction of $\mathrm{Au}(\mathrm{III})$ was attained after $200 \mathrm{~min}$ (seen by monitoring of the peak at $500 \mathrm{~nm}$ ).

Results obtained by TEM after 200 min reaction for a 1-layer cast film on a copper grid showed well-organized Au particles, with a particle diameter of approximately $3 \mathrm{~nm}$ and a narrow size distribution (as shown in the inset of Figure 1a). In addition, 
X-ray diffraction of PAMAM-Au cast films (not shown) enabled easy identification of the (111), (200), and (220) atomic planes of the Au nanoparticles. To confirm Au encapsulation inside the PAMAM dendrimer, we compared FTIR spectra in the transmission mode for neat PAMAM and PAMAM-Au in Figure $1 \mathrm{~b}$. The band at $1449 \mathrm{~cm}^{-1}$, assigned to the amide band II from inside the PAMAM molecule, splits into two bands, indicating a strong perturbation of the amide by the presence of the nanoparticles. The split indicates that the amide group can be strongly attached to the gold nanoparticle surface. Furthermore, a new shoulder appears at ca. $1665 \mathrm{~cm}^{-1}$ for PAMAM-Au, also confirming the interaction and Au encapsulation inside the PAMAM cavities. Similar results have been reported in the literature. ${ }^{25,26}$

Kinetics of PAMAM-Au Adsorption and LbL Properties. After nanoparticle formation, PAMAM-Au was assembled together with PVS onto ITO substrates in a layer-by-layer fashion. The Au-oxide reduction current was determined as a function of immersion time in PAMAM-Au using cyclic voltammetry, to ascertain the time required for total coverage of the ITO electrode surface previously covered with a PVS layer. The results are shown in Figure 2a. Voltammograms from $\mathrm{Au}$ nanoparticles in sulfuric acid solution presented a characteristic oxidation peak at $+1.2 \mathrm{~V}(\mathrm{vs} \mathrm{Ag} / \mathrm{AgCl}$ ) and a single reduction peak at $+1.00 \mathrm{~V}$, which was attributed to the reduction of $\mathrm{Au}$ oxides at the nanoparticle surface. Figure $2 \mathrm{~b}$ shows a nonlinear correlation between the electrode immersion time in the PAMAM-Au solution and the cathodic peak current. The kinetics of adsorption of PAMAM-Au LbL films can be described by a first-order equation

$$
I=I_{\max }\left[1-\exp \left(-t / t_{1}\right)\right]
$$

where $I$ is the peak current at time $t$, and $I_{\max }$ is the maximum current at infinite time. During the first 5 min of adsorption, the amount of PAMAM-Au deposited represents $95 \%$ of the total current, with no significant increase in the current being observed after this time. It is worth mentioning that the growth of multilayers in LbL films has been studied by UV-vis spectrophotometry and two deposition stages are generally identified, corresponding to fast nucleation followed by slow diffusional growth. ${ }^{29}$

The sequential deposition of the PVS/PAMAM-Au in order to obtain a multilayer assembly on the ITO substrate was carried out by immersing the ITO electrodes for $5 \mathrm{~min}$ into the PVS and PAMAM-Au solutions, alternately. The deposition of the multilayers was monitored via cyclic voltammetry, as shown in Figure 2c that features a linear increase of the anodic and cathodic peak current, indicating that the same amount of material is adsorbed during each deposition step. Using UVvis spectroscopy (not shown), we obtained similar results. Here, we can consider the system ITO-PVS/PAMAM-Au as a polymer-modified electrode, more specifically as a redox polymer system, ${ }^{31}$ which can transport charge from the electrode substrate to the external solution or vice versa. The mechanisms for charge transport within a redox polymer are electron hopping and ion migration. ${ }^{30}$ The oxidation-reduction of gold entails two different charge transport mechanisms. Electrons must flow between the nanoparticles and the electrode and the ions released by the water splitting to form the oxide must diffuse to keep electroneutrality inside the film. The limiting transport in the case of the PVS/PAMAM-Au multilayer system seems to be electron hopping, since Au-nanoparticles are immobilized inside the PAMAM molecules. This is in good agreement with the work by Laurent and Schlenoff, ${ }^{31}$ which demonstrates that
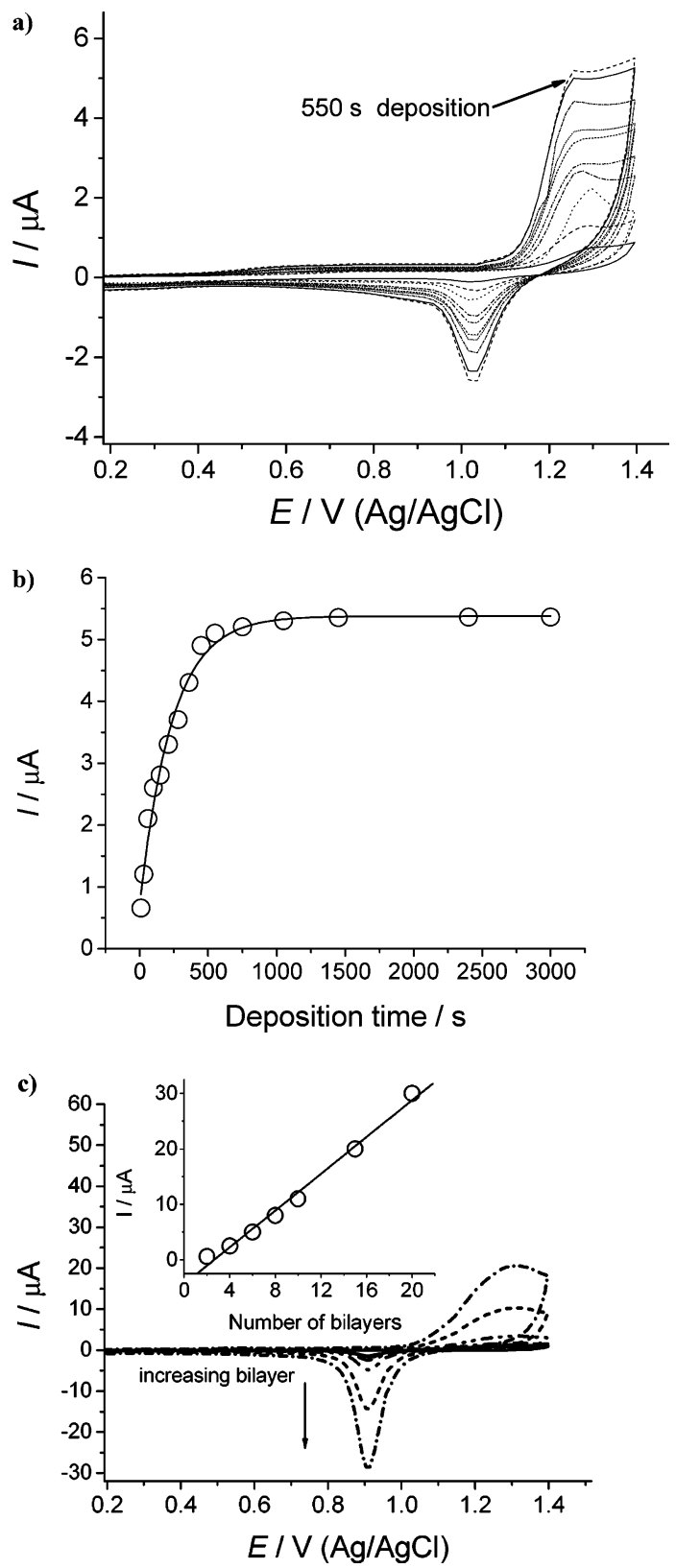

Figure 2. (a) Kinetic study using cyclic voltammetry to determine the time necessary for the total recovery of the ITO electrode surface $\left(1 \mathrm{~cm}^{2}\right)$, details in the Experimental Section. (b) Plot of gold oxidation current as a function of immersion time in PAMAM-Au. (c) Cyclic voltammograms of ITO-(PVS/PAMAM-Au $)_{n}$ with different numbers of bilayers $(n)$ and dependence of the oxidation peak current on the number of bilayers (inset). Scan rate: $50 \mathrm{mV} \mathrm{s}^{-1}$. Electrolyte solution: $0.5 \mathrm{~mol} \mathrm{~L}^{-1} \mathrm{H}_{2} \mathrm{SO}_{4}$.

electrochemically active polyelectrolytes can be incorporated in multilayer structures, in such a way that the redox-active material throughout the multilayer is electrochemically addressable via electron hopping between neighboring sites. ${ }^{31}$

One important feature in Figure 2 is that the electrochemical reactions take place at the gold nanoparticle surface, as revealed by the shape of the anodic and cathodic peaks, which are characteristic of gold surface oxides. ${ }^{30}$ The latter is evidence that the Au nanoparticles are accessible and participating in the electrochemical reactions. This fact will be further evidenced in the formation of a Prussian blue film on the nanoparticles (see below). The potential corresponding to the anodic and cathodic current peak changes linearly with the scan rate, as shown in Figure 3 for a 6-bilayer PVS/PAMAM-Au film, 


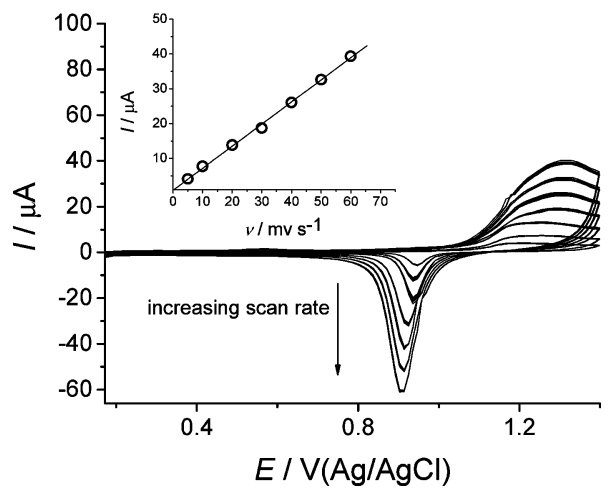

Figure 3. Influence of scan rate $(v: 5,10,20,30,40,50$, and $60 \mathrm{mV}$ $\mathrm{s}^{-1}$ ) on oxidation and reduction peak current of a 6-bilayer ITO-PVS/ PAMAM-Au modified electrode. There are 10 cycles shown for each rate. Electrolyte solution: $0.5 \mathrm{~mol} \mathrm{~L}^{-1} \mathrm{H}_{2} \mathrm{SO}_{4}$.

indicating charge transport involving the PVS/PAMAM-Au film. The PVS/PAMAM-Au LbL films were found to be very stable, with the peak current remaining the same even after many cycles. It was also observed that the films retained their electrochemical properties even after having been stored for several months at room temperature.

Charge Transport Mechanism and Kinetics. To examine the electrochemical performance of ITO-PVS/PAMAM-Au electrodes, we employed cyclic voltammetry under the same conditions as described above, in the presence of hexacyanoferrate(III) as redox marker for the electrochemical reaction at the surface of electrode and for monitoring the kinetics of charge transport. Figure 4a shows a typical voltammogram for an ITO electrode and an ITO electrode covered with a 6-bilayer PVS/ PAMAM-Au film in hexacyanoferrate(III) in $0.5 \mathrm{~mol} \mathrm{~L}^{-1} \mathrm{H}_{2}$ $\mathrm{SO}_{4}$ electrolyte solution. The voltammograms correspond to a reversible system, since $\left|I_{\mathrm{pa}} / I_{\mathrm{pc}}\right|=1$ and $\Delta E=E_{\mathrm{pa}}-E_{\mathrm{pc}}=$ $57 / n \mathrm{mV} .{ }^{32}$ Furthermore, the difference in peak potential of $57 / n$ $\mathrm{mV}$ is consistent with a semi-infinite linear diffusion, not with surface confined species for which the peak potentials should coincide. This was confirmed by varying the scan rate, $v$, as shown in Figure $4 b$, where charge transfer is diffusion-limited up to $200 \mathrm{mV} \mathrm{s}^{-1}$ at a bare ITO electrode, above which there is a lack of linearity in the $I$ versus $v^{1 / 2}$ plot. Furthermore, $\Delta E$ increases gradually with the scan rate due to a positive increase in the potential of the anodic peak at high scan rates, indicating slower kinetics. For example, a difference of $10 \mathrm{mV}$ exists between $E_{\mathrm{pa}}$ at scan rate $10 \mathrm{mV} \mathrm{s}^{-1}$ with $200 \mathrm{mV} \mathrm{s}^{-1}$ for bare ITO. On the other hand, when an ITO-PVS/PAMAM-Au electrode was employed (Figure 4c), linearity was observed up to $1500 \mathrm{mV} \mathrm{s}^{-1}$, confirming that the presence of the $\mathrm{Au}$ nanoparticles enhances the charge-transfer rate. Also, $\Delta E$ remained the same for all scan rates, as expected for reversible systems. It is worth mentioning that the increase in peak potential difference with scan rate may be due to uncompensated resistance rather than a quasireversible electron transfer. In this case, the role of Au may be to lower the ohmic (not chargetransfer) resistance of the assembly.

As shown in Figure 4, peak currents for hexacyanoferrate(II)/(III) increase linearly with the square root of the potential scan rate, from 10 to $200 \mathrm{mV} \mathrm{s}^{-1}$ for bare ITO and from 10 to $200 \mathrm{mV} \mathrm{s}^{-1}$ for 6-bilayer ITO-PVS/ PAMAM-Au. Thus, both systems show linear diffusion conditions, and the RandlesSevcik relation ${ }^{32}$ for a one-electron reaction can be applied. The influence of the nanoparticles on the electrochemical performance can be assessed by calculating the hexacyanoferrate(II)
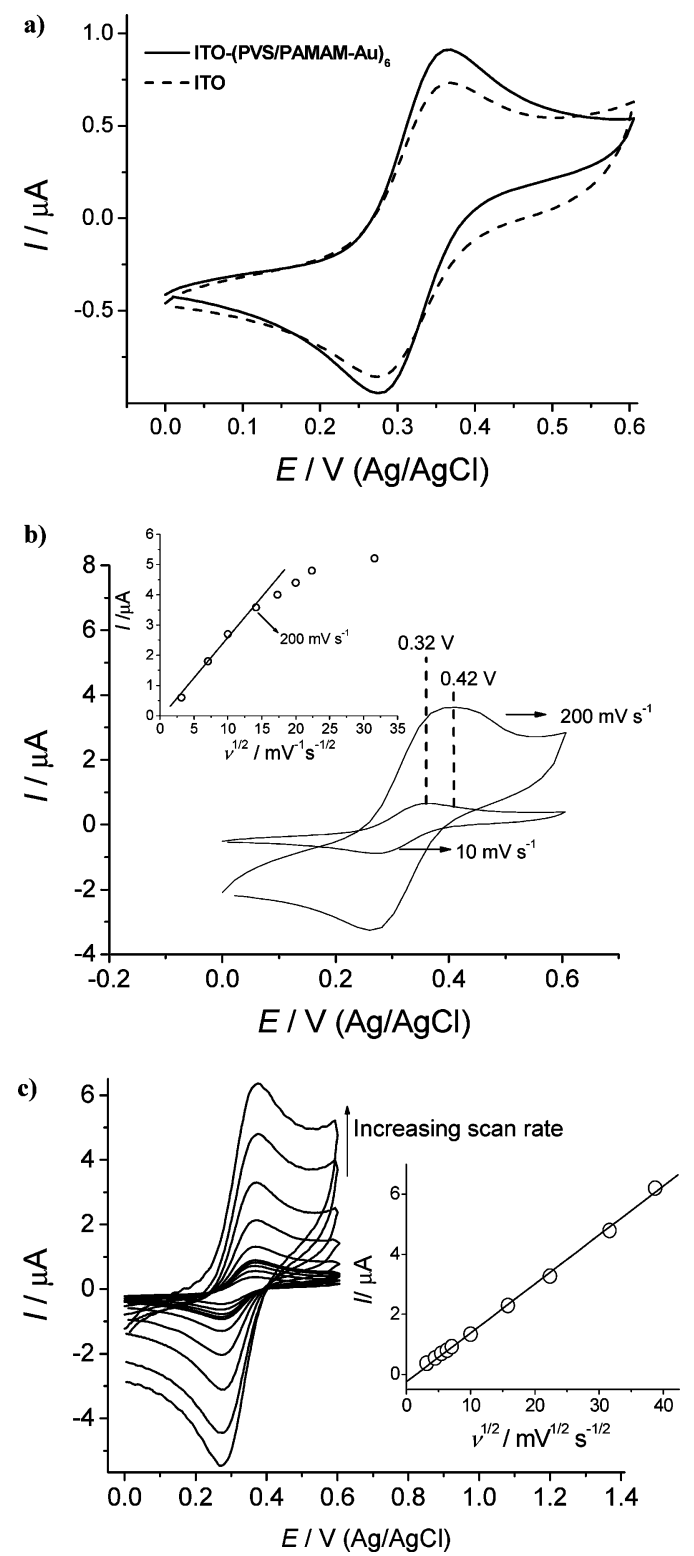

Figure 4. (a) Cyclic voltammograms for ITO and ITO covered with 6 bilayers of PVS/PAMAM-Au in the presence of $5.0 \mathrm{mmol} \mathrm{L}^{-1}$ hexacyanoferrate(III) in $0.5 \mathrm{~mol} \mathrm{~L}^{-1} \mathrm{H}_{2} \mathrm{SO}_{4}$. (b) Influence of scan rate (10 and $200 \mathrm{mV} \mathrm{s}^{-1}$ ) on oxidation and reduction peak current for 5 mmol L ${ }^{-1}$ hexacyanoferrate(III) in $0.5 \mathrm{~mol} \mathrm{~L}^{-1} \mathrm{H}_{2} \mathrm{SO}_{4}$ at an ITO electrode. Inset shows a plot of anodic peak current as a function of the square root of the scan rate. (c) Influence of scan rate on oxidation and reduction peak current for $5 \mathrm{mmol} \mathrm{L} \mathrm{L}^{-1}$ hexacyanoferrate(III) in $0.5 \mathrm{~mol} \mathrm{~L}^{-1} \mathrm{H}_{2} \mathrm{SO}_{4}$ at a 6-bilayer ITO-PVS/PAMAM-Au electrode. Inset shows a plot of the anodic peak current as a function of the square root of the scan rate.

apparent diffusion coefficient from the Randles-Sevcik relation

$$
I_{\mathrm{p}}=\left(2.687 \times 10^{5}\right) n^{3 / 2} v^{1 / 2} D_{\text {app }}{ }^{1 / 2} A C
$$

where $I_{\mathrm{p}}$ is the peak current, $n$ is the number of electrons transferred, $v$ is the scan rate $\left(\mathrm{V} \mathrm{s}^{-1}\right), A$ is the electrode area $\left(\mathrm{cm}^{2}\right), D_{\text {app }}$ is the hexacyanoferrate(II) apparent diffusion coefficient $\left(\mathrm{cm}^{2} \mathrm{~s}^{-1}\right)$, and $C$ is the hexacyanoferrate(II) concentration $\left(\mathrm{mol} \mathrm{cm}^{-3}\right)$. Under the same experimental conditions, $D_{\text {app }}$ for the hexacyanoferrate species is $4.9 \times 10^{-6}$ and $5.6 \times$ $10^{-6} \mathrm{~cm}^{2} \mathrm{~s}^{-1}$ for bare ITO and 6-bilayer ITO-PVS/ PAMAM$\mathrm{Au}$, respectively. These values appear to indicate that $\mathrm{Au}$ nanoparticles enhance the physical transport of the hexacyano- 
a)
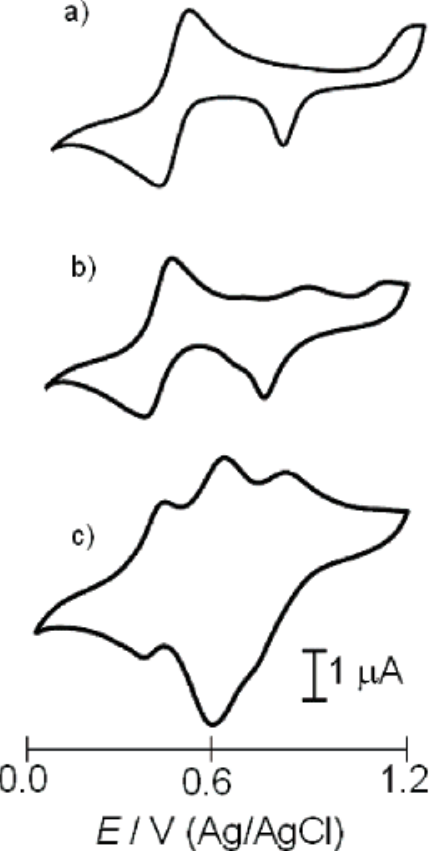

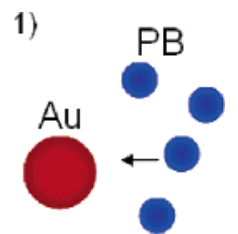

2)

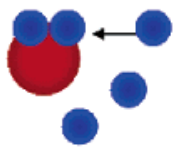

1)

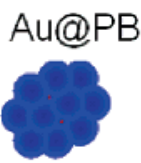

Figure 5. Cyclic voltammograms at three stages of formation of $\mathrm{Au} @ \mathrm{~PB}$ on a 6-bilayer ITO-PVS/PAMAM-Au electrode in a solution of $5.0 \mathrm{mmol} \mathrm{L}{ }^{-1}$ hexacyanoferrate(III) in $0.5 \mathrm{~mol} \mathrm{~L}^{-1} \mathrm{H}_{2} \mathrm{SO}_{4}$, scan rate $50 \mathrm{mV} \mathrm{s}^{-1}$. (a) First cycle. (b) Tenth cycle. (c) Cyclic voltammogram after $2 \mathrm{~h}$ at open circuit in the same solution, with stirring.

ferrate species. They cannot be taken as proof of the enhanced transport because the effective concentration of hexacyanoferrate in the film could be different from that of the bulk, and may be a function of film property. Further quantitative studies are required to ensure that the transport was enhanced.

Using Prussian Blue as Electrochemical Mediator. Prussian blue (PB) was employed to evaluate the adsorption of a redox mediator onto the Au-nanoparticles, via electrodeposition. The presence of the PB mediator on the nanoparticles is interesting due to its outstanding electrochemical and electrochromic properties. In addition, materials based on PB molecules have been studied in self-organized media mainly because of PB's ferromagnetic properties. ${ }^{33,34}$ Recent attempts have shown that direct assembly of ionic species onto polyelectrolytes using the LbL approach leads to ferromagnetic properties. ${ }^{35}$ Also, PB is one of the most used redox mediators in biosensor fabrication. ${ }^{37}$

The same hexacyanoferrate(III) solution described earlier was used to adsorb PB on PAMAM-Au nanoparticles. Figure 5 shows the voltammograms corresponding to three stages of formation of the system core-shell (Au@PB). During the first potential cycle in Figure 5a, a redox pair appears due to hexacyanoferrate(II)/(III), in addition to the oxidation and reduction peaks of the gold nanoparticles. This suggests that the gold surface is electroactive, with no formation of PB. After 10 cycles, the gold nanoparticles' surface is already partially covered by adsorbed $\mathrm{PB}$, as shown by the presence of two new anodic peaks attributable to $\mathrm{PB}$ at ca. 0.6 and $0.8 \mathrm{~V}$, together with the peak at $1.2 \mathrm{~V}$ due to the gold surface (Figure $5 \mathrm{~b}$ ). Following these 10 potential cycles, the electrode was left for more than $2 \mathrm{~h}$ in the same solution, with stirring, at open circuit. After this time, the gold oxidation peaks were no longer observed, and the cyclic voltammograms of the PVS/PAMAMAu modified electrode (Figure 5c) revealed the well-known form of the reversible reduction and oxidation of $\mathrm{PB} .{ }^{37}$ This indicates that the PB film had completely covered the $\mathrm{Au}$ nanoparticle surface, with formation of an ITO-PVS/PAMAM-Au@PB electrode.

It may be envisaged that this approach can be explored in technological applications where a redox mediator is required to act in a highly efficient, effective way.

\section{Conclusions}

Electrochemical and spectroscopic studies have been undertaken to better understand PAMAM-Au multilayers formed by the LbL technique. The deposition of individual PAMAM-Au layers has been examined in detail: the adsorption kinetics was determined by cyclic voltammetry to be first-order, and $5 \mathrm{~min}$ of adsorption was sufficient for maximum coverage. Formation of PVS/PAMAM-Au multilayers showed a linear increase in anodic and cathodic peak currents, indicating that the same amount of material was adsorbed in each deposition step. Electron-hopping was considered as the charge transport mechanism between PAMAM-Au layers.

It was also shown that the charge transport in the ITO-PVS/ PAMAM-Au system was faster than that for a bare ITO electrode. With hexacyanoferrate(III) to probe the electrochemical reaction at the electrode surface, it could be inferred that the charge transport in the PAMAM-Au layers was faster than that for nonmodified electrodes. A new system based on PAMAM-Au@PB was prepared by simple potential cycling electrodeposition after ITO-PVS/PAMAM-Au LbL film preparation. The latter approach can be applied in a number of technological applications such as biosensors and nanoelectronics where a reversible redox mediator is required.

Acknowledgment. Financial support from FAPESP, Capes (process number 1238/05-1), CNPq, IMMP/MCT (Brazil), and Fundação para a Ciência e Tecnologia (FCT) Portugal, ICEMS (Research Unit 103), is acknowledged. The authors are also indebted to Madalina Maria Barsan for helpful discussions.

\section{References and Notes}

(1) Zhao, M. Q.; Crooks, R. M. Adv. Mater. 1999, 11, 217.

(2) Zhao, M. Q.; Crooks, R. M. Chem. Mater. 1999, 11, 3379

(3) Tomalia, D. A.; Berry, V.; Hall, M.; Hedstrand, D. M. Macromolecules 1987, 20, 1164.

(4) Crooks, R. M.; Zhao, M. Q.; Sun, L.; Chechik, V.; Yeung, L. K. Top. Curr. Chem. 2001, 212, 81 .

(5) Balogh, L.; Laverdure, K. S.; Gido, S. P.; Mott, A. G.; Miller, M. J.; Ketchel, B. P.; Tomalia, D. A. Mater. Res. Soc. Symp. Proc. 1999, 756, 69

(6) He, J. A.; Valluzzi, R.; Yang, K.; Dolukhanyan, T.; Sung, C. M. Kumar, J.; Tripathy, S. K.; Samuelson, L.; Balogh, L.; Tomalia, D. A. Chem. Mater. 1999, 11, 3268.

(7) Balogh, L.; Valluzzi, R.; Hagnauer, G. L.; Laverdure, K. S.; Gido, S. P.; Tomalia, D. A. J. Nanopart. Res. 1999, 1 (3), 353.

(8) Esumi, K.; Hosoya, T.; Suzuki, A.; Torigoe, K. Langmuir 2000, 16,2978

(9) Sui, G.; Mabrouki, M.; Ma, Y.; Micic, M.; Leblanc R. M. J. Colloid Interface Sci. 2002, 250, 364

(10) Cheng, L.; Cox, J. A. Electrochem Commun. 2001, 3, 285.

(11) Suzdalev, I. P.; Suzdalev, P. I. Russ. Chem. Rev. 2001, 70, 177.

(12) Rao, C. N. R.; Kulkarni, G. U.; Thomas, P. J.; Edwards, P. P. Chem. Eur. J. 2002, 8, 29.

(13) Quinn, B. M.; Liljeroth, P.; Ruiz, V.; Laaksonen, T.; Kontturi, K J. Am. Chem. Soc. 2003, 125, 6644.

(14) Chen, S.; Ingram, R. S.; Hostetler, M. J.; Pietron, J. J.; Murray, R. W.; Schaaff, T. G.; Khoury, J. T.; Alvarez, M. M.; Whetten, R. L. Science 1998, 280, 2098.

(15) Crespilho, F. N.; Huguenin, F.; Zucolotto, V.; Olivi, P.; Nart, F. C.; Oliveira, O. N., Jr. Electrochem. Commun. 2006, 8, 348.

(16) Valden, M.; Lai, X.; Goodman, D. W. Science 1998, 281, 1647.

(17) Wang, J.; Xu, D.; Kawde, A.-N.; Polsky, R. Anal. Chem. 2001, 73,5576 .

(18) Li, Y.; Boone, E.; El-Sayed, M. A. Langmuir 2002, 18, 4921.

(19) Kastner, M. A. Rev. Mod. Phys. 1992, 64, 849.

(20) Kötz, R.; Carlen, M. Electrochim. Acta 2000, 45, 2483. 
(21) Jensen, T. R.; Malinsky, M. D.; Haynes, C. L.; Van Duyne, R. P. J. Phys. Chem. B 2000, 104, 10549.

(22) Kim, Y.-G.; Oh, S.-K.; Crooks, R. M.; Chem. Mater. 2004, 16, 167.

(23) Dai, X.; Nekrassova, O.; Hyde, M. E.; Compton, R. G. Anal. Chem. 2004, 76, 5924 .

(24) Mandal, B. K.; Suzuki, K. T. Talanta 2002, 58, 201.

(25) Esumi, K.; Nakamura, R.; Suzuki, A.; Torigoe, K.; Langmuir 2000, 16,7842 .

(26) Yang, L.; Luo, Y.; Jia, X.; Ji, Y.; You, L.; Zhou, Q. J. Phys. Chem. B 2004, 108, 1176 .

(27) Crespilho, F. N.; Zucolotto, V.; Siqueira, J. R., Jr., Constantino, C. J. L.; Nart, F. C.; Oliveira, O. N., Jr. Environ. Sci. Technol. 2005, 39 , 5385.

(28) Zucolotto, V.; Ferreira, M.; Cordeiro, M. R.; Constantino, C. J. L.; Balogh, D. T.; Zanatta, A. R.; Moreira, W. C.; Oliveira, O. N., Jr. J. Phys. Chem. B 2003, 107, 3733.
(29) Raposo, M.; Pontes, R. S.; Mattoso, L. H. C.; Oliveira, O. N., Jr. Macromolecules 1997, 30, 6095 .

(30) Bard A. J.; Faulkner, L. R. Electrochemical Methods: Fundamentals and Applications; Wiley: New York, 1980.

(31) Laurent, D.; Schlenoff, J. B. Langmuir 1997, 13, 1552.

(32) Brett, C. M. A. Electrochemistry: Principles, Methods and Applications; Oxford University Press: Oxford, 1993.

(33) Mingotaud, C.; Lafuente, C.; Amiell, J.; Delhaes, P. Langmuir 1999, 15,289

(34) Jaiswal, A.; Colins, J.; Agricole, B.; Delhaes, P.; Ravaine, S. J. Colloid Interface Sci. 2003, 261, 330.

(35) Pyrasch, M.; Tieke, B.; Langmuir 2001, 17, 7706.

(36) Laurent, D.; Schlenoff, J. B. Langmuir 1997, 13, 1552.

(37) Ricci, F.; Palleschi, G. Biosens. Bioelectron. 2005, 21, 389. 